\title{
Photoisomerization reactions of 4-methoxy- and 4-hydroxy-6-methyl- o-pyrones: An experimental matrix isolation and theoretical density functional theory study $\dagger$
}

\author{
Susana Breda, ${ }^{a b}$ Leszek Lapinski, ${ }^{a}$ Rui Fausto ${ }^{b}$ and Maciej J. Nowak ${ }^{* a}$ \\ ${ }^{a}$ Institute of Physics, Polish Academy of Sciences, Al. Lotnikow 32/46, 02-668, Warsaw, \\ Poland.E-mail: mjnow@ifpan.edu.pl \\ ${ }^{b}$ Department of Chemistry, University of Coimbra, 3004-535, Coimbra, Portugal
}

Received 17th July 2003, Accepted 2nd September 2003

First published as an Advance Article on the web 19th September 2003

\begin{abstract}
Photoisomerization reactions were studied for 4-methoxy- and 4-hydroxy-6-methyl- $\alpha$-pyrones isolated in lowtemperature Ar matrices. Two types of photoproducts: Dewar valence isomer and conjugated aldehyde-ketene were photogenerated upon UV irradiation of the matrix-isolated compounds. After prolonged irradiation practically all the initial material was converted to the corresponding Dewar isomers, whereas the population of aldehyde-ketene photoproducts was marginal. The structure of the main photoproducts was positively identified by comparison of their experimental IR spectra with the spectra theoretically predicted for the Dewar isomers. Good agreement between the experimental observations and the theoretical calculations allowed for reliable assignment of the infrared bands in the spectra of the initial forms of the studied compounds as well as in the spectra of the Dewar isomers. For the 4-hydroxy-6-methyl- $\alpha$-pyrone the presence of its minor tautomeric form in a low-temperature matrix was detected.
\end{abstract}

\section{Introduction}

Photochemistry of $\alpha$-pyrones has been the subject of intensive study since the early work of de Mayo, ${ }^{1}$ who reported an openring ester photoproduct generated by UV irradiation of methanolic solution of 4,6-dimethyl- $\alpha$-pyrone. Conjugated aldehyde-ketene was proposed as the directly produced intermediate of this reaction, but no proof of the existence of this species was provided. On the other hand, Corey and Streith ${ }^{2}$ observed (by ${ }^{1} \mathrm{H}$ NMR spectroscopy) creation of the Dewar valence isomer of $\alpha$-pyrone in the photochemical study of the compound dissolved in ether. In the study of Guthrie et al. ${ }^{3}$ where thin films of solid 4,6-dimethyl- $\alpha$-pyrone or 4-methoxy-6-methyl$\alpha$-pyrone were UV irradiated (through Pyrex), infrared bands attributable to Dewar valence isomers were observed at $c a$ $1810 \mathrm{~cm}^{-1}$.

Matrix isolation investigations on the photochemistry of $\alpha$-pyrones, carried out so far, concerned practically exclusively the unsubstituted parent compound. For $\alpha$-pyrone isolated in an Ar matrix, rapid formation of aldehyde-ketene photoproduct (revealed by the appearance of intense IR band at 2146 $\mathrm{cm}^{-1}$ ) was reported. ${ }^{4,5}$ The Dewar valence isomer was also created, under these conditions, but at a much lower rate. The

\footnotetext{
$\dagger$ Electronic supplementary information (ESI) available: Tables S1, S3 and S6 provide internal coordinates used in the normal mode analysis. Atom numbering is given in Scheme S1. The assignment of the experimental spectra of 4-methoxy- and 4-hydroxy-6-methyl- $\alpha$-pyrones is provided in Tables S2 and S7. The assignment of the experimental spectra of the photoproducts generated from 4-methoxy- and 4-hydroxy-6-methyl- $\alpha$-pyrones is given in Tables S4 and S10. The theoretically calculated spectra of isomer IV of 4-methoxy-6-methyl- $\alpha$-pyrone and isomers $\mathbf{V}$ and VI of 4-hydroxy-6-methyl- $\alpha$-pyrone are given in Tables S5, S8 and S9, respectively. Comparison of the experimental spectrum recorded after UV $(\lambda>320 \mathrm{~nm})$ irradiation of matrix isolated 4-hydroxy-6-methyl- $\alpha$-pyrone with the spectra theoretically calculated for Dewar isomers VIII and IX is presented in Fig. S1. See http://www.rsc.org/suppdata/cp/b3/b308116h/
}

conclusion about the photogeneration of this photoproduct was based on observation of the new band at $1850 \mathrm{~cm}^{-1}$ in the IR spectrum of the UV irradiated matrix. The photoreactions leading to the Dewar valence isomer and openring ketene were recently studied for 4,6-dimethyl- $\alpha$-pyrone isolated in Ar matrix. ${ }^{6}$

In the present work, photochemical reactions of matrixisolated 4-methoxy- and 4-hydroxy-6-methyl- $\alpha$-pyrones were investigated. Photoproduced aldehyde-ketene and Dewar isomer were observed after UV irradiation of low-temperature matrices with isolated monomers of the compounds in question. Contrary to the case of the $\alpha$-pyrone itself, for the currently investigated substituted $\alpha$-pyrones, the effective isomerization to the Dewar form was found to be the dominating photoprocess. Dewar photoproducts were identified on the basis of comparison of their experimental IR spectra with the results of theoretical simulations carried out at the DFT(B3LYP)/6-311++G(d,p) level.

\section{Experimental}

4-Methoxy-6-methyl- $\alpha$-pyrone and 4-hydroxy-6-methyl- $\alpha$-pyrone used in this study were commercial products supplied by Aldrich. The samples were heated in a microoven placed in the vacuum chamber of a cryostat. The vapors of the studied compounds were deposited, together with a large excess of argon (of spectral purity, Linde AG), on the CsI window cooled to $10 \mathrm{~K}$. The IR spectra were recorded with $0.5 \mathrm{~cm}^{-1}$ resolution using Thermo Nicolet Nexus 670 FTIR spectrometer equipped with a $\mathrm{KBr}$ beamsplitter and a DTGS detector. Integral intensities of the IR absorption bands were measured by numerical integration. Matrices were irradiated with the light from a high pressure mercury lamp HBO 200 fitted with a water filter and cut-off filters transmitting light with $\lambda>320$ $\mathrm{nm}$ or $\lambda>270 \mathrm{~nm}$.

Phys. Chem. Chem. Phys., 2003, 5, 4527-4532 


\section{Computational}

The equilibrium geometries of the 4-methoxy- and 4-hydroxy6-methyl- $\alpha$-pyrone isomers were fully optimized at the DFT(B3LYP)/6-311++G(d,p) level of theory. ${ }^{7,8}$ Subsequently, the harmonic wavenumbers were calculated at the optimized geometries using the same theoretical method. All calculations were carried out using the GAUSSIAN 98 program. ${ }^{9}$

Transformations of the force constant matrices in Cartesian coordinates to the molecule-fixed internal coordinates allowed the ordinary normal-coordinate analysis to be performed as described by Schachtschneider. ${ }^{10}$ The internal coordinates used in this analysis (listed in Tables S1, S3 and S6 of the ESI $\dagger$ ) were defined in the manner recommended by Pulay et al. ${ }^{11}$ Potential energy distribution (PED) matrices $^{12}$ have been calculated and the elements of these matrices greater than $10 \%$ are given in Tables S2, S4, S5 and S7-S10. $\dagger$

In order to correct for vibrational anharmonicity, basis set truncation and the neglected part of electron correlation, the calculated DFT wavenumbers were scaled down by a single factor of 0.98 .

Electronic energies of 4-hydroxy-6-methyl- $\alpha$-pyrone tautomers were also calculated at the MP2 level, at geometries optimized using this method.

\section{Results and discussion}

\subsection{Photochemistry of 4-methoxy-6-methyl- $\alpha$-pyrone}

The IR spectrum of 4-methoxy-6-methyl- $\alpha$-pyrone isolated in Ar matrix is presented in Fig. 1. This spectrum is compared with the results of the theoretical calculations of the normal mode frequencies and IR intensities performed for structure I (Scheme 1) of this compound at the DFT(B3LYP)/6$311++\mathrm{G}(\mathrm{d}, \mathrm{p})$ level. Very good agreement between the experimentally observed and theoretically calculated spectra allowed a reliable assignment of the IR bands. Experimental and theoretical frequencies, IR intensities and the potential energy distribution (PED) of the corresponding normal modes are collected in Table S2. $\dagger$ Three characteristic bands due to the stretching vibrations of the three double bonds in the molecule $(\mathrm{C}=\mathrm{O}, \mathrm{C} 5=\mathrm{C} 6, \mathrm{C} 3=\mathrm{C} 4)$ appear in the experimental spectrum at 1757,1664 and $1582 \mathrm{~cm}^{-1}$, respectively. Analogous bands were previously observed ${ }^{13}$ for unsubstituted $\alpha$-pyrone at frequencies: 1781,1646 and $1557 \mathrm{~cm}^{-1}$. The isomer of 4-methoxy-6-methyl- $\alpha$-pyrone with the methoxy group rotated by $180^{\circ}$ is energetically less stable (by $13 \mathrm{~kJ} \mathrm{~mol}^{-1}$, DFT) and

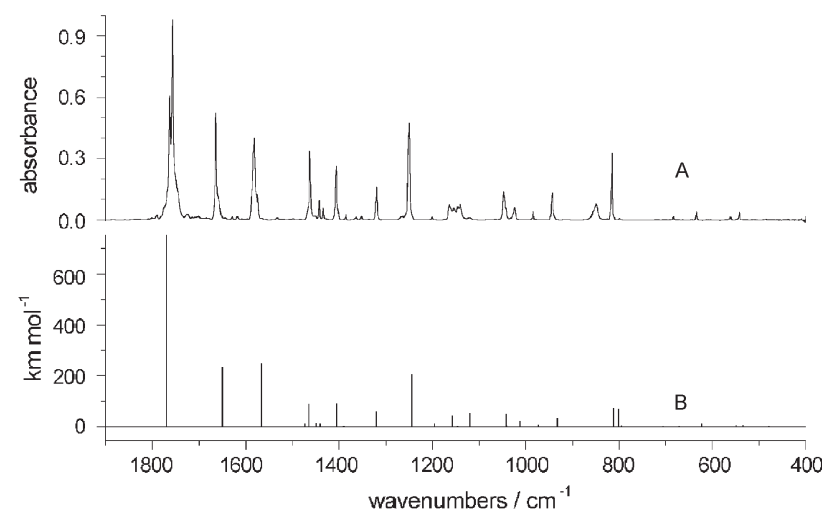

Fig. 1 (A) Infrared spectrum of 4-methoxy-6-methyl- $\alpha$-pyrone isolated in Ar matrix; (B) the spectrum of the form $\mathbf{I}$ of the compound theoretically predicted at the DFT(B3LYP)/6-311++G(d,p) level. Theoretical wavenumbers were scaled by 0.98 . The baseline of the experimental spectrum was corrected.<smiles>COc1ccc(=O)oc1</smiles>

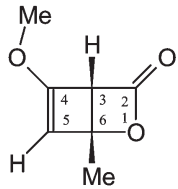

III<smiles>CO/C=C\C(=O)OC</smiles>

II

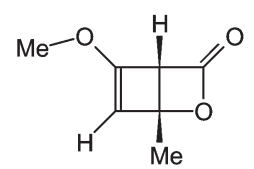

IV
Scheme 1 Isomers of 4-methoxy-6-methyl- $\alpha$-pyrone.

its theoretically calculated IR spectrum does not match the experimental observation.

$\mathrm{UV}(\lambda>320 \mathrm{~nm})$ irradiation of matrix isolated 4-methoxy6-methyl- $\alpha$-pyrone resulted in a decrease of the initial IR spectrum and the appearance of new bands due to photoproducts. The strong band at $1828 \mathrm{~cm}^{-1}$ indicates the formation of the Dewar valence isomer, ${ }^{4-6}$ whereas the weak band at 2149 / $2146 \mathrm{~cm}^{-1}$ can be assigned to the aldehyde-ketene II. During the progress of the photoreaction (presented in Fig. 2) the initial form of the compound was continuously converted to the Dewar isomer. The population of the aldehyde-ketene photoproduct was very low at any stage of the reaction, as can be concluded from the low intensity of the $2149 / 2146$ $\mathrm{cm}^{-1}$ band. This band is due to the "antisymmetric" stretching vibration of the ketene $\mathrm{C}=\mathrm{C}=\mathrm{O}$ group. Such bands are known to be very intense and their theoretically predicted absolute intensities are higher than $1000 \mathrm{~km} \mathrm{~mol}^{-1}$. Hence, the observed low relative intensity of this band (Fig. 2) is a

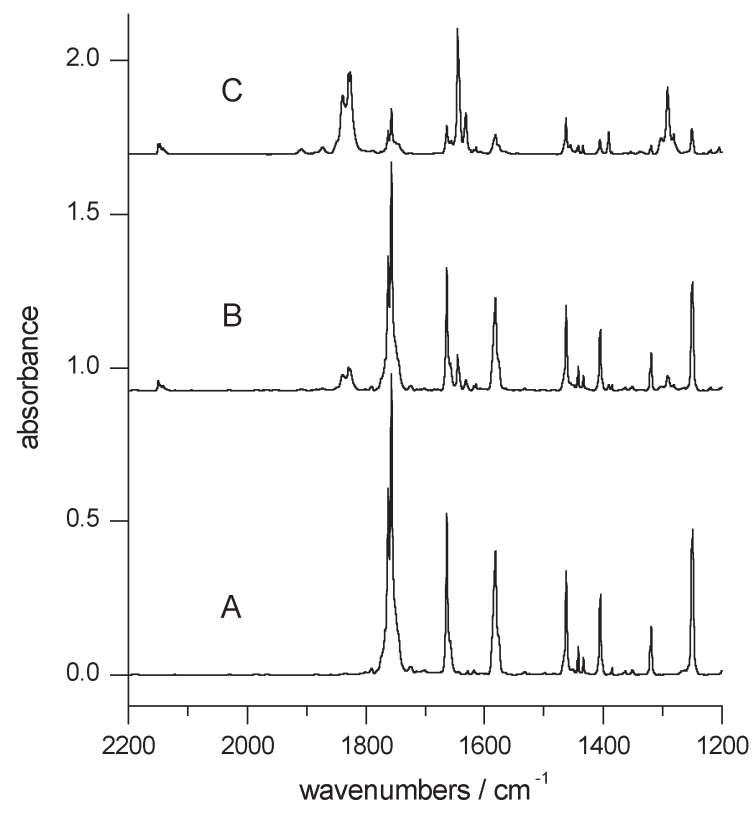

Fig. 2 Progress of the photoreaction occurring for 4-methoxy-6methyl- $\alpha$-pyrone monomers upon UV irradiation; (A) portion of the IR spectrum recorded after deposition of the Ar matrix; (B) after 45 min of UV $(\lambda>320 \mathrm{~nm})$ irradiation; (C) after $8 \mathrm{~h}$ of UV $(\lambda>320$ $\mathrm{nm}$ ) irradiation. Growth of the IR band at $1828 \mathrm{~cm}^{-1}$ indicates creation of the Dewar isomer; the band at $2149 / 2146 \mathrm{~cm}^{-1}$ is due to open-ring aldehyde-ketene. Baselines of the experimental spectra were corrected. 
clear indication of very low efficiency of the ring opening reaction for matrix isolated 4-methoxy-6-methyl- $\alpha$-pyrone.

On the basis of the series of IR spectra recorded at different stages of UV irradiation it was possible to identify all the bands belonging to the spectrum of the main photoproduct. This spectrum is presented in Fig. 3 and compared with the spectrum calculated (at the DFT(B3LYP)/6-311++G(d,p) level) for the optimized structure of the Dewar valence isomer III. The frequencies and intensities of the IR bands observed in the spectrum are compared in Table $\mathrm{S} 4 \dagger$ with the corresponding theoretical values. The proposed assignment of the IR spectrum is also given in this table. The agreement between the experimental and theoretical spectra is good enough to leave practically no doubt about the correctness of the assignment of the photoproduct to the Dewar structure.

In the Dewar form of 4-methoxy-6-methyl- $\alpha$-pyrone there are only two double bonds and, accordingly, two bands due to their stretching vibrations were found in the range 1900$1500 \mathrm{~cm}^{-1}$ of the IR spectrum. The first absorption at 1839/ $1828 \mathrm{~cm}^{-1}$ is due to the $\nu(\mathrm{C}=\mathrm{O})$ vibration. As it is usually the case, ${ }^{14,15}$ the frequency of the $\mathrm{C}=\mathrm{O}$ stretching vibration is significantly higher for the carbonyl group attached to a four-membered ring than for the group attached to a six-membered ring. The band at $1645 / 1632 \mathrm{~cm}^{-1}$ should be assigned to the stretching vibration of the only ring double bond $\mathrm{C} 4=\mathrm{C} 5$. Both bands mentioned above are split in two components. This feature could be an indication of the photoproduction of two rotamers III and IV of the Dewar isomer. The theoretically predicted IR spectra corresponding to these two structures are very similar (compare Tables S4 and S5 in the $\mathrm{ESI}+$ ). Nevertheless, the fact that the pattern of the split $\nu(\mathrm{C}=\mathrm{O})$ and $\nu(\mathrm{C} 4=\mathrm{C} 5)$ bands is well reproduced by the superposition of the theoretical spectra of the two rotamers (Fig. 4) suggests that both forms are stabilized in the low-temperature matrix after UV irradiation.

\subsection{Photochemistry of 4-hydroxy-6-methyl- $\alpha$-pyrone}

The infrared spectrum of 4-hydroxy-6-methyl- $\alpha$-pyrone isolated in Ar matrix is presented in Fig. 5 and compared with the spectrum of form $\mathbf{V}$ (Scheme 2), theoretically simulated at the DFT(B3LYP)/6-311++G(d,p) level. Experimental and theoretical frequencies, IR intensities and the potential energy distribution (PED) of the corresponding normal modes are collected in Table S7. $\dagger$ Several characteristic bands were observed in this spectrum. The band due to the stretching vibration of the $\mathrm{OH}$ group was found at $3614 \mathrm{~cm}^{-1}$ and the

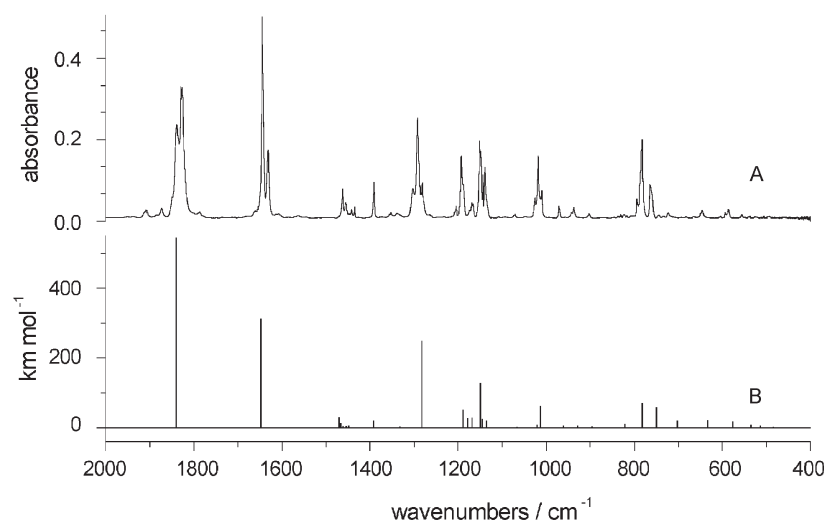

Fig. 3 Infrared spectrum of the photoproduced Dewar valence isomer of 4-methoxy-6-methyl- $\alpha$-pyrone: (A) experimental spectrum of the species generated upon UV $(\lambda>320 \mathrm{~nm})$ irradiation of matrix isolated 4-methoxy-6-methyl- $\alpha$-pyrone; (B) the spectrum of the Dewar isomer III theoretically predicted at DFT(B3LYP)/6-311++G(d,p) level. Theoretical wavenumbers were scaled by 0.98 . Baseline of the experimental spectrum was corrected.

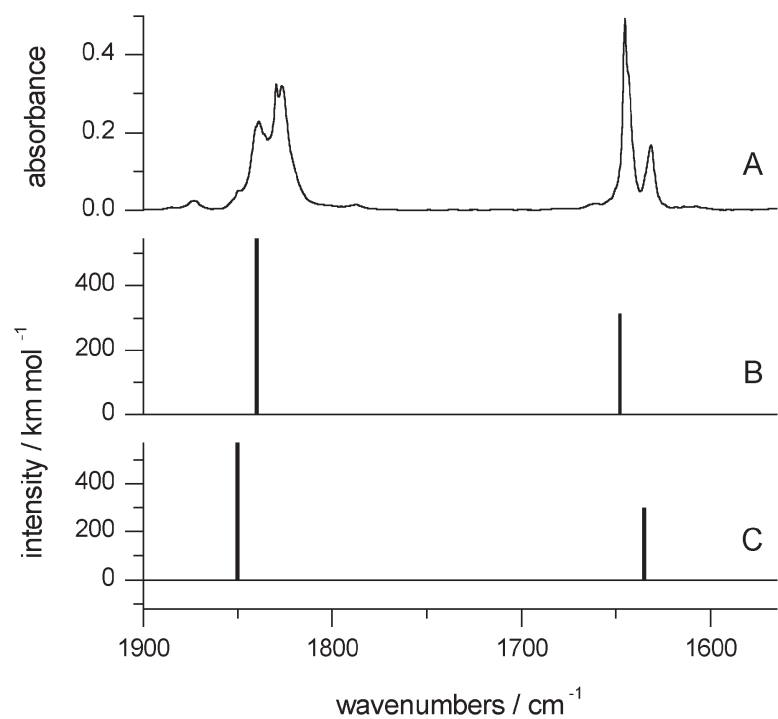

Fig. 4 (A) Portion of the infrared spectrum of the photoproduct generated upon UV $(\lambda>320 \mathrm{~nm})$ irradiation of matrix isolated 4-methoxy-6-methyl- $\alpha$-pyrone; (B) corresponding portion of the theoretical DFT(B3LYP)/6-311++G(d,p) spectrum of the Dewar isomer in its rotameric form III; $(\mathrm{C})$ in the rotameric form IV.

band due to the torsional vibration of this group at $441 /$ $438 / 428 \mathrm{~cm}^{-1}$. The three bands corresponding to the stretching vibrations of the double bonds, $\mathrm{C}=\mathrm{O}, \mathrm{C} 5=\mathrm{C} 6$ and $\mathrm{C} 3=\mathrm{C} 4$, were observed at frequencies: 1766,1665 and 1591 $\mathrm{cm}^{-1}$, very similar to the spectral positions of their counterparts in the spectrum of the methoxy derivative. In the spectrum of 4-hydroxy-6-methyl- $\alpha$-pyrone the spectral region $1850-1550 \mathrm{~cm}^{-1}$, where the bands due to stretching vibrations of double bonds are usually observed, is complicated by the appearance of some additional bands at 1814/1802 and $1705 / 1699 \mathrm{~cm}^{-1}$. These bands (of medium/weak relative intensities) can not be easily explained by comparing the experimental spectrum with the spectrum calculated for the form $\mathbf{V}$ (Fig. 5). This point will be discussed in more detail in the forthcoming section.

Matrix-isolated monomers of 4-hydroxy-6-methyl- $\alpha$-pyrone were irradiated with UV light $(\lambda>320 \mathrm{~nm})$. The effects of this irradiation (see Fig. 6) were similar to those observed in the
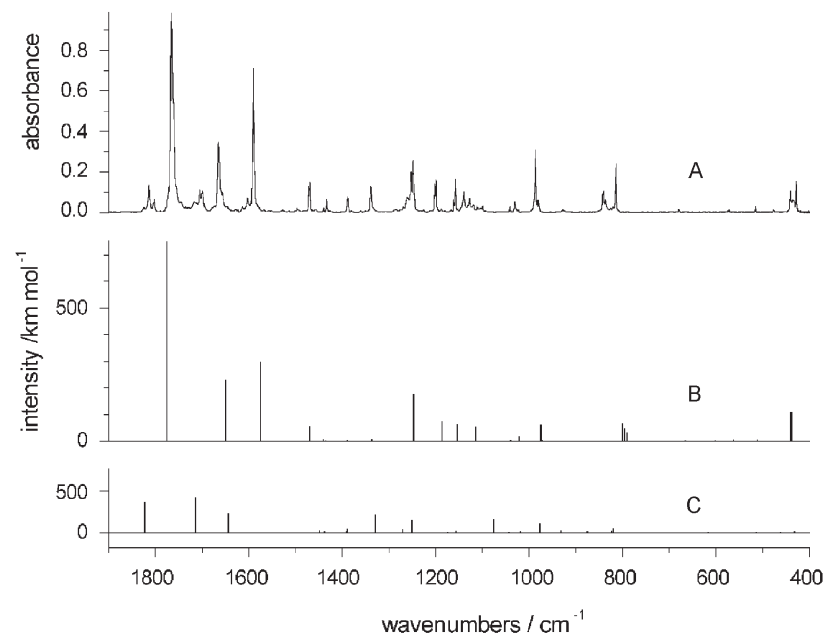

Fig. 5 (A) Infrared spectrum of 4-hydroxy-6-methyl- $\alpha$-pyrone isolated in $\mathrm{Ar}$ matrix; (B) the spectrum theoretically predicted (at DFT(B3LYP)/6-311++G(d,p) level) for the tautomer $\mathbf{V}$ of the compound; (C) theoretical spectrum of the tautomer VI. Theoretical wavenumbers were scaled by 0.98 . Baseline of the experimental spectrum was corrected. 
<smiles>Cc1oc(=O)ccc1O</smiles>

V

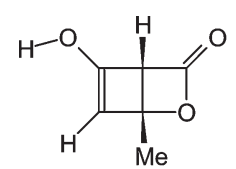

VIII<smiles>CC1=CC(=O)CC(C)OC1=O</smiles>

VI

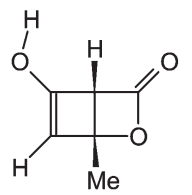

IX<smiles>Cc1cc(=O)cc(O)o1</smiles>

VII
Scheme 2 Isomers of 4-hydroxy-6-methyl- $\alpha$-pyrone.

case of the methoxy derivative. Creation of small amounts of the open-ring ketene was revealed by the band at $2147 \mathrm{~cm}^{-1}$. The most intense band growing in the spectrum of the main photoproduct appeared at $1838 \mathrm{~cm}^{-1}$. Such a characteristic frequency ${ }^{4-6}$ strongly suggested that the band is due to the $\mathrm{C}=\mathrm{O}$ stretching vibration of the Dewar isomer. The spectral

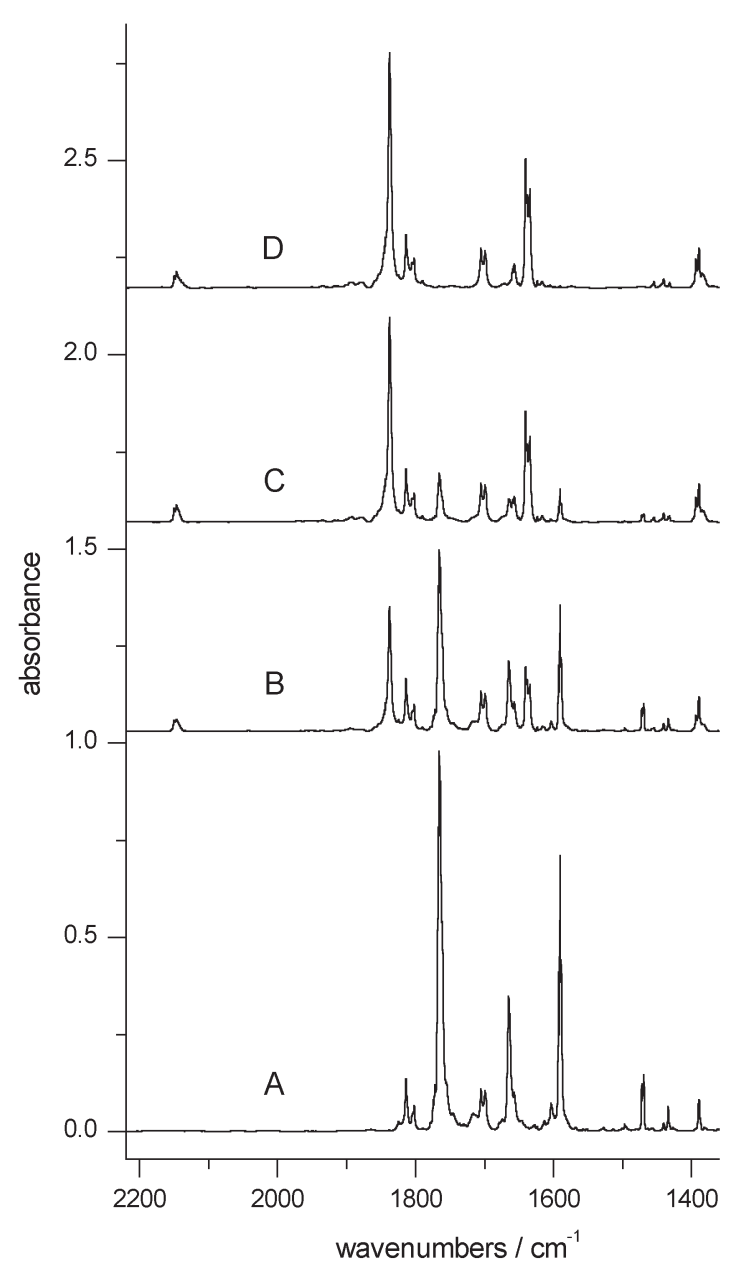

Fig. 6 Progress of the photoreaction occurring for 4-hydroxy-6methyl- $\alpha$-pyrone monomers upon UV irradiation; (A) portion of the IR spectrum recorded after deposition of the Ar matrix; (B) after 10 min of UV $(\lambda>320 \mathrm{~nm})$ irradiation; (C) after $30 \mathrm{~min}$ of UV $(\lambda>320 \mathrm{~nm})$ irradiation, (D) after $2 \mathrm{~h}$ of $\mathrm{UV}(\lambda>320 \mathrm{~nm})$ irradiation. Growth of the IR band at $1838 \mathrm{~cm}^{-1}$ indicates creation of the Dewar isomer; the band at $2147 \mathrm{~cm}^{-1}$ is due to open-ring aldehydeketene. Baselines of the experimental spectra were corrected. position of this band matches well that of the analogous absorption observed for the Dewar isomer of the methoxy derivative. In addition, also the band at $1641 / 1634 \mathrm{~cm}^{-1}$, ascribable to the $\nu(\mathrm{C} 4=\mathrm{C} 5)$ vibration, appears at a frequency similar to that of the corresponding absorption of the Dewar form of 4-methoxy-6-methyl- $\alpha$-pyrone. These bands, as well as the general pattern of the spectrum recorded after UV $(\lambda>320 \mathrm{~nm})$ irradiation, are well reproduced by the spectrum theoretically predicted for the Dewar valence isomer in its rotameric form VIII (see Fig. 7 traces A, B and Table S10†). The calculated spectrum of the second rotamer IX of the Dewar form differs significantly (Fig. S1 $\dagger$ ) from that predicted for the structure VIII and does not reproduce the experimental spectrum presented in Fig. 7 trace A. Hence, one can conclude that the form VIII is the main photoproduct generated from 4-hydroxy-6-methyl- $\alpha$-pyrone upon UV $(\lambda>320 \mathrm{~nm})$ irradiation.

The frequency of the $\nu(\mathrm{OH})$ band in the spectrum of the Dewar isomer $\left(3608 \mathrm{~cm}^{-1}\right.$; Fig. 8 trace B) differs little from that of the analogous vibration in the initial form $\mathbf{V}$ (3614 $\mathrm{cm}^{-1}$; Fig. 8 trace A). This observation is in agreement with the theoretical calculations that predict the frequency of the $\nu(\mathrm{OH})$ band in the spectrum of 4-hydroxy-6-methyl- $\alpha$-pyrone $\mathbf{V}$ to be only slightly higher than the position of the corresponding band in the spectrum of the Dewar form VIII (see Tables S7 and S10 $\dagger$ ).

Not all the bands observed in the initial spectrum of 4-hydroxy-6-methyl- $\alpha$-pyrone decreased upon UV $(\lambda>320$ $\mathrm{nm})$ irradiation. The above mentioned bands at $1814 / 1802$ and $1705 / 1699 \mathrm{~cm}^{-1}$, as well as a set of other bands did not change upon exposure to $\mathrm{UV}(\lambda>320 \mathrm{~nm})$ radiation (see Fig. 9 traces $\mathrm{A}$ and B). On the other hand, the intensities of the bands from this set decreased significantly (Fig. 9 traces $\mathrm{B}$ and $\mathrm{C}$ ), when the matrix was additionally irradiated with the UV $(\lambda>270 \mathrm{~nm})$ light. No intensity changes upon UV $(\lambda>270 \mathrm{~nm})$ irradiation were observed for the bands in the spectrum of the photoproduced Dewar isomer. It is noteworthy that the $3700-3500 \mathrm{~cm}^{-1}$ region of the IR spectrum, where the bands due to $\nu(\mathrm{OH})$ vibrations are observed, was to the smallest detail the same before and after UV $(\lambda>270$ $\mathrm{nm}$ ) irradiation (Fig. 8 traces B and C). The observations described above demonstrate the presence of a second chemical species in the matrix immediately after its deposition. Obvious candidates for this species are tautomeric forms VI

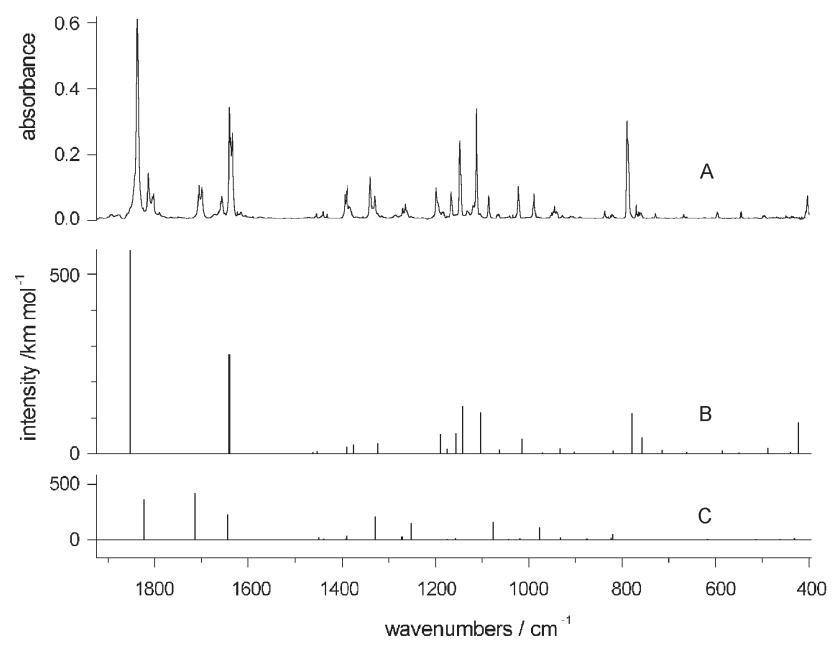

Fig. 7 (A) Experimental spectrum recorded after UV $(\lambda>320 \mathrm{~nm})$ irradiation of matrix isolated 4-hydroxy-6-methyl- $\alpha$-pyrone; (B) the spectrum of the Dewar isomer VIII theoretically predicted at DFT(B3LYP)/6-311++G(d,p) level; (C) theoretical spectrum of the tautomer VI. Theoretical wavenumbers were scaled by 0.98 . Baseline of the experimental spectrum was corrected. 


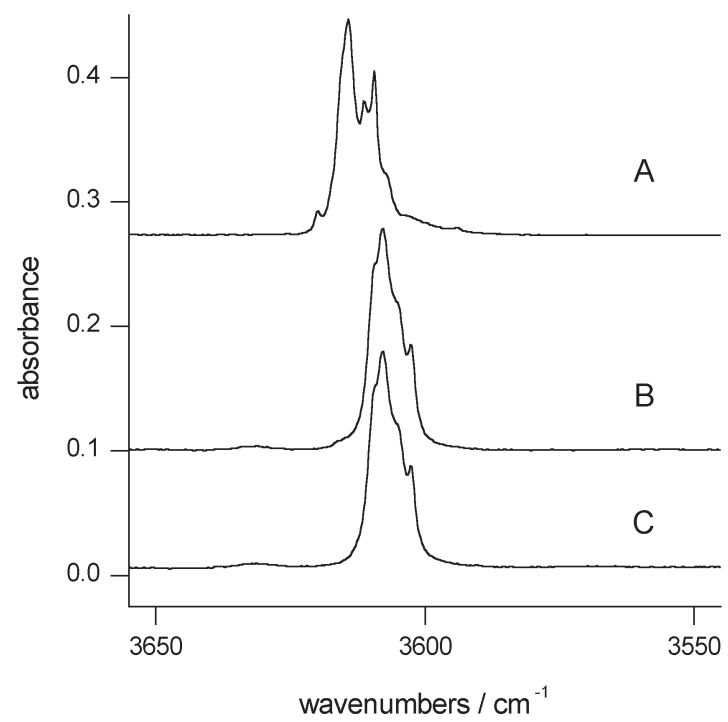

Fig. 8 The $\nu(\mathrm{OH})$ range of the IR spectrum recorded: (A) after deposition of the Ar matrix isolated 4-hydroxy-6-methyl- $\alpha$-pyrone; (B) after UV $(\lambda>320 \mathrm{~nm})$ irradiation; (C) after subsequent UV $(\lambda>270 \mathrm{~nm})$ irradiation.

and VII of 4-hydroxy-6-methyl- $\alpha$-pyrone shown in the Scheme 2. There are several arguments in favor of the assignment of the less populated form of 4-hydroxy-6-methyl- $\alpha$-pyrone as the tautomer VI. First, the theoretically calculated energy of this form is not much different from that of the form $\mathbf{V}$. Considering the values presented in Table 1 one has to remember that relative energies of tautomers calculated at the applied levels of theory are usually accurate within $\pm 10 \mathrm{~kJ} \mathrm{~mol}^{-1}$ limits. ${ }^{16}$ The calculated energy of the tautomer VII is significantly ( $c$ a $40 \mathrm{~kJ} \mathrm{~mol}^{-1}$ ) higher with respect to the form $\mathbf{V}$; that

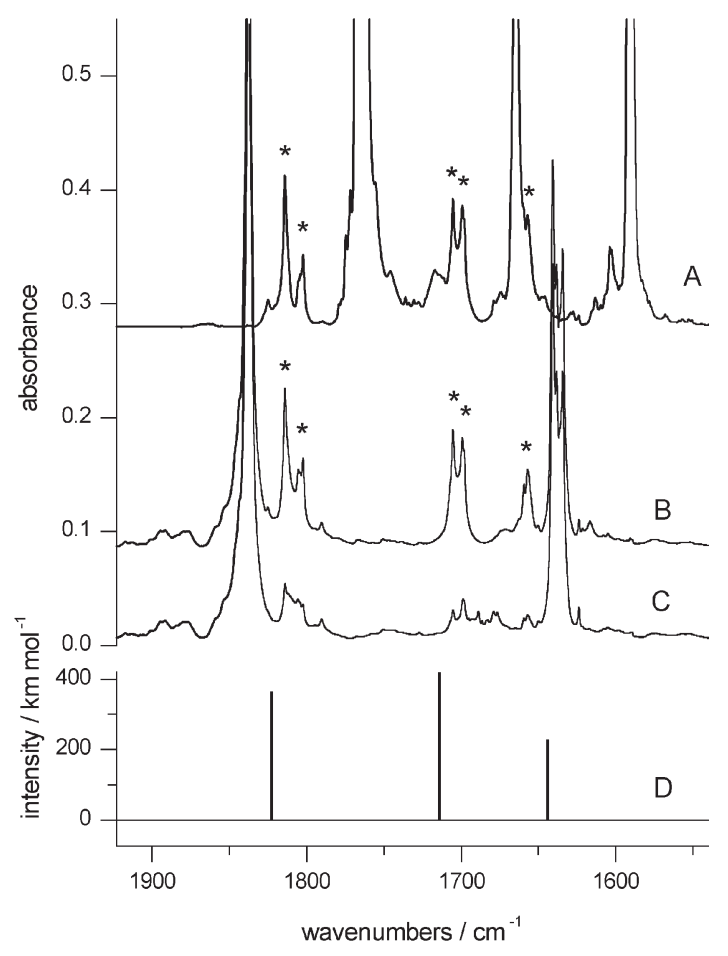

Fig. 9 Portion of the IR spectrum of Ar matrix isolated 4-hydroxy-6methyl- $\alpha$-pyrone recorded: (A) after deposition; (B) after UV $(\lambda>320$ $\mathrm{nm})$ irradiation; (C) after subsequent UV $(\lambda>270 \mathrm{~nm})$ irradiation; (D) theoretical spectrum of the tautomer VI. Theoretical wavenumbers were scaled by 0.98 .
Table 1 Relative energies (in $\mathrm{kJ} \mathrm{mol}^{-1}$ ) of 4-hydroxy-6-methyl- $\alpha$ pyrone isomers $^{a}$

\begin{tabular}{lrrrr}
\hline Energies & \multicolumn{1}{c}{ VI } & VII & VIII & IX \\
\hline$\Delta E_{\mathrm{el}}(\mathrm{DFT})^{b}$ & 7.0 & 38.6 & 193.9 & 195.4 \\
$\Delta E_{\mathrm{el}}(\mathrm{MP} 2)^{c}$ & -5.7 & 40.2 & & \\
$\Delta E_{\mathrm{ZPE}}(\mathrm{DFT})^{b}$ & 1.7 & 0.5 & & \\
$\Delta E_{\mathrm{Tot}}(\mathrm{DFT})^{d}$ & 8.7 & 39.1 & & \\
$\Delta E_{\mathrm{Tot}}(\mathrm{MP} 2)^{e}$ & -4.0 & 40.7 & &
\end{tabular}

${ }^{a}$ Relative to form $\mathbf{V}^{b}$ Electronic and zero point (ZPE) energy calculated at DFT(B3LYP)/6-311++G(d,p) level. ${ }^{c}$ Electronic energy calculated at MP2/6-31G(d,p) level. ${ }^{d} \Delta E_{\mathrm{Tot}}(\mathrm{DFT})=\Delta E_{\mathrm{el}}(\mathrm{DFT})+$ $\Delta E_{\mathrm{ZPE}}(\mathrm{DFT}) .{ }^{e} \Delta E_{\mathrm{Tot}}(\mathrm{MP} 2)=\Delta E_{\mathrm{el}}(\mathrm{MP} 2)+\Delta E_{\mathrm{ZPE}}(\mathrm{DFT})$

makes the observation of the form VII very improbable. Second, the absence of any observable change in the 3700-3500 $\mathrm{cm}^{-1}$ region of the spectrum upon UV $(\lambda>270 \mathrm{~nm})$ irradiation, while all the bands due to the minor isomer decreased significantly, strongly suggests that there is no $\mathrm{OH}$ group in this isomer. Finally, the whole set of experimental bands, which (on the basis of their behavior by UV $(\lambda>270 \mathrm{~nm})$ irradiation) can be assigned to the minor isomer of 4-hydroxy-6methyl- $\alpha$-pyrone, is well reproduced by the spectrum theoretically calculated for structure VI (Tables S7 and S9†, and traces $\mathrm{C}$ in Figs. 5 and 7). Especially convincing is the comparison of the observed and calculated spectral patterns in the 1850-1550 $\mathrm{cm}^{-1}$ region, where the bands due to stretching vibrations of double bonds are expected to appear. All three bands due to the stretching vibrations of the two $\mathrm{C}=\mathrm{O}$ groups and of the $\mathrm{C} 5=\mathrm{C} 6$ bond are clearly observed (Fig. $9 \mathrm{~A}$ and $\mathrm{B}$ ) at positions $\left(1814 / 1802,1705 / 1699\right.$ and $\left.1657 \mathrm{~cm}^{-1}\right)$, which agree well with the theoretical predictions $\left(1823,1714\right.$ and $\left.1644 \mathrm{~cm}^{-1}\right)$. The relative population of tautomers $\mathbf{V}$ and VI in a low-temperature matrix was estimated as a ratio of the sum of integral intensities of the bands at 1766, 1665 and $1591 \mathrm{~cm}^{-1}$ (from V) and the sum of integral intensities of the bands at 1814/ $1802,1705 / 1699$ and $1657 \mathrm{~cm}^{-1}$ (form VI) scaled by the respective theoretical absolute intensities calculated at the DFT level. As a result the $[\mathbf{V}]:[\mathbf{V I}]$ ratio equal to 4.3 was obtained.

Proton exchange leading to $\mathbf{V} \leftrightarrow \mathbf{V I}$ tautomerism can be quick and effective, in spite of the fact that hydrogen atoms bound to the ring carbon atoms are usually not labile. The position of the $\mathrm{CH}_{2}$ group between the two electron withdrawing $\mathrm{C}=\mathrm{O}$ groups is similar to the position of the $\mathrm{CH}_{2}$ group in malonates. The wealth of malonate chemistry is based, in significant part, on the lability of the $\mathrm{CH}_{2}$ group hydrogens. ${ }^{17}$ Hence, by analogy, the $\mathrm{CH}_{2}$ protons in the form VI can also be labile and the ratio of tautomers $\mathbf{V}$ and $\mathbf{V I}$ trapped in a low-temperature matrix can correspond to the gas-phase equilibrium.

\subsection{Comparison with the photochemistry of unsubstituted a-pyrone}

The photochemical behavior of unsubstituted $\alpha$-pyrone isolated in $\mathrm{Ar}$ matrices ${ }^{4,5}$ and the photochemistry of matrixisolated 4-methoxy- and 4-hydroxy-6-methyl- $\alpha$-pyrones, investigated in the present work, differ strikingly in the relative effectiveness of the two competing processes: $\alpha$-bond cleavage and valence isomerization to the Dewar form. In the case of $\alpha$-pyrone itself, the Norrish type I reaction leading to conjugated aldehyde-ketene was previously observed ${ }^{4,5}$ as a vigorous reaction transforming significant quantities of the compound to the open-ring isomer, whereas the Dewar form was created very slowly. For the compounds studied in the current work, similarly as for 4,6-dimethyl- $\alpha$-pyrone, ${ }^{6}$ the population of the open-ring ketene was very low at any stage of the photoprocess. 
These substituent-related effects could be rationalized as follows. The Norrish type I, $\alpha$-bond cleavage reaction is commonly believed ${ }^{18-21}$ to originate from the first excited $n \pi^{*}$ state, while the valence isomerization to the Dewar form should occur starting from an excited state with $\pi \pi^{*}$ character. In compounds like $\alpha$-pyrone, the lowest excited singlet state $\mathrm{S}_{1}$ has always $n \pi^{*}$ character, but the energy gap between this state and the $\mathrm{S}_{2} \pi \pi^{*}$ state depends on the substituents attached to the $\alpha$-pyrone ring. Conjugative type substitution with groups such as $-\mathrm{OH},-\mathrm{CH}_{3}$ or $-\mathrm{OCH}_{3}$, should blue-shift the $\mathrm{S}_{0} \rightarrow \mathrm{S}_{1}\left(\mathrm{n} \pi^{*}\right)$ transition and red-shift the $\mathrm{S}_{0} \rightarrow \mathrm{S}_{2}\left(\pi \pi^{*}\right)$ transition. ${ }^{22}$ Hence, the gap between the $S_{1}$ and $S_{2}$ states should be reduced for $\alpha$-pyrones substituted with such groups with respect to the $\alpha$-pyrone itself. The spectral shifts described above were recently experimentally observed for $\alpha$-pyrone, 4-methoxy-6-methyl- $\alpha$-pyrone and 4-hydroxy-6-methyl- $\alpha$ pyrone dissolved in cyclohexane or ethanol. ${ }^{23}$

The Norrish type $\mathrm{I}$ reaction in $\mathrm{S}_{1}$ is not a barrierless process $^{20}$ and some excess of excitation energy is needed to promote the $\alpha$-bond cleavage. Hence, this process does not necessarily need to dominate, even though the lowest of the excited states is of the $n \pi^{*}$ character. If the energy gap between the $n \pi^{*}$ and $\pi \pi^{*}$ states is not too large, the processes typical for $\pi \pi^{*}$ photochemistry can compete with the $\alpha$-bond cleavage or even dominate. Isomerizations to the Dewar forms, observed as dominating processes for 4-hydroxy- and 4-methoxy-6methyl- $\alpha$-pyrones, are good examples of such behavior.

The influence of conjugative type substitution on the relative effectiveness of $\alpha$-bond cleavage and Dewar isomer formation was also previously studied for $4(3 \mathrm{H})$-pyrimidinones isolated in low-temperature inert gas matrices. ${ }^{14}$ The conclusions of the last study were the same as those resulting from the present observations: with an increasing number of conjugative type substituents the valence isomerization to the Dewar form was promoted and the $\alpha$-cleavage reaction was hindered.

\section{Conclusions}

The photoisomerization reactions of 4-methoxy- and 4hydroxy-6-methyl- $\alpha$-pyrones to the corresponding Dewar valence isomers were observed for the compounds isolated in low-temperature Ar matrices. Comparison of the experimental IR spectra recorded after UV irradiation of the matrices with the theoretical spectra, calculated for the Dewar forms of the compounds in question, allowed a reliable identification of the photoproduced species. Conjugated aldehyde-ketenes were also produced during UV irradiation of the investigated $\alpha$ pyrones, but the population of these open-ring species was found to be very low at any stage of the photoreaction.

\section{Acknowledgements}

SB acknowledges support from the Centre of Excellence ASPECT financed by the European Commission within the contract G6MA-CT-2002-04021. The SB and RF contribution to this work was also run under the POCTI/UI/43366/2001 project, which is partially supported by FEDER.

\section{References}

1 P. de Mayo, Adv. Org. Chem., 1960, 2, 394

2 E. J. Corey and J. Streith, J. Am. Chem. Soc., 1964, 86, 950.

3 J. P. Guthrie, C. L. McIntosh and P. de Mayo, Can. J. Chem., 1970, 48, 237.

4 O. L. Chapman, C. L. McIntosh and J. P. Pacansky, J. Am. Chem. Soc., 1973, 95, 244.

5 R. G. S. Pong and J. S. Shirk, J. Am. Chem. Soc., 1973 , 95, 248.

6 S. Breda, L. Lapinski, I. Reva and R. Fausto, J. Photochem. Photobiol. A, 2003, in press.

7 A. D. Becke, Phys. Rev. A, 1988, 38, 3098.

8 C. T. Lee, W. T. Yang and R. G. Parr, Phys. Rev. B, 1988, 37, 785 .

9 M. J. Frisch, G. W. Trucks, H. B. Schlegel, G. E. Scuseria, M. A. Robb, J. R. Cheeseman, V. G. Zakrzewski, J. A. Montgomery, Jr., R. E. Stratmann, J. C. Burant, S. Dapprich, J. M. Millam, A. D. Daniels, K. N. Kudin, M. C. Strain, O. Farkas, J. Tomasi, V. Barone, M. Cossi, R. Cammi, B. Mennucci, C. Pomelli, C. Adamo, S. Clifford, J. Ochterski, G. A. Petersson, P. Y. Ayala, Q. Cui, K. Morokuma, D. K. Malick, A. D. Rabuck, K. Raghavachari, J. B. Foresman, J. Cioslowski, J. V. Ortiz, A. G. Baboul, B. B. Stefanov, G. Liu, A. Liashenko, P. Piskorz, I. Komaromi, R. Gomperts, R. L. Martin, D. J. Fox, T. Keith, M. A. Al-Laham, C. Y. Peng, A. Nanayakkara, M. Challacombe, P. M. W. Gill, B. Johnson, W. Chen, M. W. Wong, J. L. Andres, C. Gonzalez, M. Head-Gordon, E. S. Replogle and J. A. Pople, Gaussian 98, Revision A.9, Gaussian, Inc., Pittsburgh PA, 1998.

10 J. H. Schachtschneider, Technical Report; Shell Development Co., Emeryville, CA, 1969.

11 P. Pulay, G. Fogarasi, F. Pang and J. E. Boggs, J. Am. Chem. Soc., 1979, 101, 2550

12 G. Keresztury and G. Jalsovszky, J. Mol. Struct., 1971, 10, 304.

13 R. Fausto, G. Quinteiro and S. Breda, J. Mol. Struct., 2001, 598 287.

14 L. Lapinski, M. J. Nowak, A. Les and L. Adamowicz, J. Am. Chem. Soc., 1994, 116, 1461.

15 H. Rostkowska, M. J. Nowak, L. Lapinski, D. Smith and L. Adamowicz, Spectrochim. Acta, Part A, 1997, 53, 959.

16 L. Lapinski, M. J. Nowak, J. Fulara, A. Les and L. Adamowicz, J. Phys. Chem., 1992, 96, 6250.

17 M. A. Aramendia, V. Borau, C. Jimenez, J. M. Marinas, J. R. Ruiz and F. J. Urbano, Tetrahedron Lett., 2002, 43, 2847.

18 N. J. Turro, Modern Molecular Photochemistry, University Science Books, Sausalito, California, 1991, ch. 7.10.

19 M. Klessinger and J. Michl, Excited States and Photochemistry of Organic Molecules, VCH Publishers, New York, 1995, ch. 7.21.

20 E. W.-G. Diau, C. Kotting and A. Zewail, ChemPhysChem., 2001, 2, 273.

21 E. W.-G. Diau, C. Kotting and A. Zewail, ChemPhysChem., 2001, 2, 294.

22 R. S. Becker, Theory and Interpretation of Fluorescence and Phosphorescence, Wiley/Interscience, New York, 1969, ch. 12.

23 J. Seixas de Melo, G. Quinteiro, J. Pina, S. Breda and R. Fausto, J. Mol. Struct., 2001, 565-566, 59. 